\title{
A CHARACTERIZATION OF NORMAL MARKOV CHAINS ${ }^{1}$
}

JAMES YACKEL

1. Introduction. The class of normal Markov chains was introduced by Kemeny and Snell [2] in their discussion of potential theory for discrete denumerable recurrent Markov chains. This class of chains is characterized here in terms of the ergodicity of an embedded semiMarkov chain. From this point of view it is shown, in terms of familiar first passage probabilities, what is required for a Markov chain to be normal.

Let $\left\{X_{n}, n \geqq 0\right\}$ be a recurrent Markov chain with denumerable state space $I$, and transition matrix $P$. We assume that $P^{n}$ approaches a limit as $n$ becomes infinite; hence the chain is either null or noncyclic ergodic. We choose a nonzero regular measure $\alpha$, cf. [3, Chapter 9], i.e. $\alpha P=\alpha$, and denote by $N_{i j}(n)$ the element in row $i$ column $j$ of the matrix $\left(I+P+P^{2}+\cdots+P^{n}\right)$.

DEFINITION 1.1. If

$$
\lim _{n \rightarrow \infty}\left[N_{i i}(n) \alpha_{j} / \alpha_{i}-N_{i j}(n)\right]
$$

and

$$
\lim _{n \rightarrow \infty}\left[N_{j j}(n)-N_{i j}(n)\right]
$$

both exist for all $i$ and $j$, then the chain is said to be normal.

We also consider a transition matrix for a dual or reversed Markov chain defined by

$$
\hat{p}_{i j}=p_{j i} \alpha_{j} / \alpha_{i} .
$$

The expression in (1) is then seen to be the expression in (2) stated for the dual chain, i.e. (1) holds iff $\hat{N}_{j j}(n)-\hat{N}_{i j}(n)$ has a limit as $n$ becomes infinite. ( $\hat{N}$ denotes the obvious quantity for the dual chain.)

2. The embedded semi-Markov chain. Let two states $i$ and $j$ be fixed throughout the discussion. We henceforth restrict ourselves to that part of the space where $X_{0}=i$ or $X_{0}=j$, then we define $Y_{n}$ $=n-\sup \left[k: X_{k} \in\{i, j\}, k \leqq n\right]$ and $Z_{n}=X_{n-Y_{n}}$.

Proposition 2.1. The two dimensional process $\left\{\left(Z_{n}, Y_{n}\right), n \geqq 0\right\}$ is a Markov chain and $\left\{Z_{n}, n \geqq 0\right\}$ is a semi-Markov chain.

Received by the editors July 20, 1967.

1 This research was supported by a National Science Foundation Contract GP 7631, with Purdue University. 
Proof. We show only that $\left(Z_{n}, Y_{n}\right)$ is a Markov chain, the assertion that $\left\{Z_{n}, n \geqq 0\right\}$ is a semi-Markov chain will then be obvious, cf. [4]. The states of the Markov chain are $(z, y), z=i, j$ and $y$ $=0,1,2, \cdots$ with transition probabilities determined by first passage taboo probabilities, cf. [1, §I, 9]. Let $d(y)=1-{ }_{j} f_{i i}(y)-{ }_{i} f_{i j}(y)$, then

$$
\begin{aligned}
p_{(i, y),(i, 0)} & ={ }_{i} f_{i i}(y+1) / d(y), \\
p_{(i, y),(j, 0)} & ={ }_{i} f_{i j}(y+1) / d(y), \\
p_{(i, y),(i, y+1)} & =d(y+1) / d(y),
\end{aligned}
$$

with similar transition probabilities for state $(j, y)$.

There is analogously defined a semi-Markov chain embedded in the dual chain, we will refer to it as $\left\{\hat{Z}_{n}, n \geqq 0\right\}$.

\section{The main theorem.}

THEOREM 3.1. The limit (2) exists if and only if the embedded semiMarkov chain $\left\{Z_{n}, n \geqq 0\right\}$ is ergodic.

COROLLARY 3.2. The chain $\left\{X_{n}, n \geqq 0\right\}$ is normal if and only if the embedded semi-Markov chains $\left\{Z_{n}, n \geqq 0\right\}$ and $\left\{\hat{Z}_{n}, n \geqq 0\right\}$ are both ergodic for all pairs $i, j$.

The proof of Corollary 3.2 is immediate upon recalling the duality of the limits (1) and (2) of Definition 1.1.

Proof of Theorem 3.1. We begin by introducing some notation. We write

$$
{ }_{i} F_{i j}(n)=\sum_{k=0}^{n}{ }_{i} f_{i j}(k)
$$

and similar expressions with appropriate subscripts to denote partial sums of first passage probabilities.

Then we see that

$$
\begin{aligned}
N_{i j}(n) & =\sum_{k=0}^{n} p_{i j}(k)=\sum_{k=0}^{n}\left\{\sum_{m=0}^{k} f_{i j}(k-m) p_{j j}(m)+\sum_{m=0}^{k}{ }_{j} f_{i i}(k-m) p_{i j}(m)\right\} \\
& =\sum_{m=0}^{n}\left\{p_{j j}(m)_{i} F_{i j}(n-m)+p_{i j}(m)_{j} F_{i i}(n-m)\right\} .
\end{aligned}
$$

To easily verify this result, reason probabilistically according to the first transition to either $i$ or $j$ whichever occurs first.

Thus we write 


$$
\begin{aligned}
N_{j j}(n)-N_{i j}(n)= & \sum_{k=0}^{n} p_{j j}(k)\left[1-{ }_{i} F_{i j}(n-k)-{ }_{j} F_{i i}(n-k)\right] \\
& +\sum_{k=0}^{n}{ }_{j} F_{i i}(n-k)\left[p_{j j}(k)-p_{i j}(k)\right] .
\end{aligned}
$$

Now decomposition of $p_{j j}$ in the first expression according to the last visit to state $i$ gives the relation

$$
p_{j j}(k)=\sum_{m=0}^{k} p_{j i}(m)_{i} p_{i j}(k-m)+{ }_{i} p_{j j}(k)
$$

and using this, (3) becomes

$$
\begin{gathered}
\sum_{k=0}^{n}\left(\sum_{m=0}^{k} p_{j i}(k-m){ }_{i} p_{i j}(m)\left[1-{ }_{i} F_{i j}(n-k)-{ }_{j} F_{i i}(n-k)\right]\right) \\
+\sum_{k=0}^{n}{ }_{i} p_{j j}(k)\left[1-{ }_{i} F_{i j}(n-k)-{ }_{j} F_{i i}(n-k)\right] \\
+\sum_{k=0}^{n}{ }_{j} F_{i i}(n-k)\left[p_{j j}(k)-p_{i j}(k)\right] .
\end{gathered}
$$

Interchanging the order of summation in the first term we can write

$$
\begin{gathered}
\sum_{m=0}^{n}{ }_{i} p_{i j}(m) \sum_{k=m}^{n} p_{j i}(k-m)\left[1-{ }_{i} F_{i j}(n-k)-{ }_{j} F_{i i}(n-k)\right] \\
=\sum_{m=0}^{n}{ }_{i} p_{i j}(m) \sum_{k=m}^{n} P\left[Z_{n-m}=i, Y_{n-m}=n-k \mid Z_{0}=j\right] \\
=\sum_{m=0}^{n}{ }_{i} p_{i j}(m) P\left[Z_{n-m}=i \mid Z_{0}=j\right] .
\end{gathered}
$$

The term (4) is $o(1)$ as $n \rightarrow \infty$, and the term (5) can be rewritten as

$$
\sum_{k=0}^{n}{ }_{j} f_{i i}(k)\left[N_{j j}(n-k)-N_{i j}(n-k)\right] .
$$

By the ergodicity of the Markov chain we know that $p_{j j}(k)-p_{i j}(k) \rightarrow 0$ as $k \rightarrow \infty$ so that $\left[N_{j j}(n)-N_{i j}(n)\right]-\left[N_{j j}(n-k)-N_{i j}(n-k)\right] \rightarrow 0$ as $n \rightarrow \infty$ and $\sum_{k} f_{i i}(k)<1$ by the recurrence of the chain so that

$$
\begin{aligned}
{\left[N_{j j}(n)-N_{i j}(n)\right]-\sum_{k=0}^{n}{ }_{j} f_{i i}(k)\left[N_{j j}(n-k)-N_{i j}(n-k)\right] } \\
=\left[1-{ }_{j} F_{i i}(\infty)\right]\left[N_{j j}(n)-N_{i j}(n)\right]+i o(1) .
\end{aligned}
$$


We conclude from (3), (6) and (7) that

$$
\begin{aligned}
{\left[N_{j j}(n)-N_{i j}(n)\right]\left[1-{ }_{j} F_{i i}(\infty)\right] } & \\
& =\sum_{m=0}^{n}{ }_{i} p_{i j}(m) P\left[Z_{n-m}=i \mid Z_{0}=j\right]+o(1)
\end{aligned}
$$

and this can be put in the form

$$
\left[N_{j j}(n)-N_{i j}(n)\right]={ }_{j} N_{i i} \sum_{m=0}^{n}{ }_{i} p_{i j}(m) P\left[Z_{n-m}=i \mid Z_{0}=j\right]+o(1) .
$$

Also we have that

$$
P\left[Z_{n}=i \mid Z_{0}=j\right]-P\left[Z_{n-k}=i \mid Z_{0}=j\right] \rightarrow 0
$$

as $n \rightarrow \infty$ whether or not $\left\{Z_{n}, n \geqq 0\right\}$ is ergodic, cf. [5]. However $\sum_{m i} p_{i j}(m)<\infty$, so that we may conclude that $N_{j j}(n)-N_{i j}(n)$ has a limit if and only if $P\left[Z_{n}=i \mid Z_{0}=j\right]$ has a limit as $n \rightarrow \infty$.

4. Ergodicity of the embedded semi-Markov chains. If the Markov chain is not null then the embedded semi-Markov chain is ergodic and hence the chain is normal according to Theorem 3.1 and its corollary. If the chain is null, then, when properly normalized, at least one of the distributions ${ }_{i} F_{i j},{ }_{j} F_{j i},{ }_{j} F_{i i}$ or ${ }_{i} F_{j j}$ does not have a finite first moment. This situation was studied in [5] and conditions on these first passage distributions are given there to insure the ergodicity of the semi-Markov chain.

The expression for the distribution of the dual semi-Markov chain $\hat{Z}$ can be written

$$
\begin{aligned}
P\left[\hat{Z}_{n}=i \mid \hat{Z}_{0}=i\right]= & \sum_{k=0}^{n} \hat{p}_{i i}(k)\left[1-{ }_{i} \hat{F}_{i j}(n-k)-{ }_{j} \hat{F}_{i i}(n-k)\right] \\
= & \sum_{k=0}^{n} p_{i i}(k)\left[1-{ }_{j} F_{j i}(n-k) \alpha_{j} / \alpha_{i}-{ }_{j} F_{i i}(n-k)\right] \\
= & P\left[Z_{n}=i \mid Z_{0}=i\right] \\
& +\sum_{k=0}^{n} p_{i i}(k)\left[{ }_{i} F_{i j}(n-k)-{ }_{j} F_{j i}(n-k) \alpha_{j} / \alpha_{i}\right] .
\end{aligned}
$$

Thus in order that both the semi-Markov and dual semi-Markov embedded chains be ergodic the distributions ${ }_{j} F_{j i}$ and ${ }_{i} F_{i j}$ must have the same type of behavior in the tails. For example, as shown in [5], it is necessary that 


$$
\int_{0}^{T}\left[{ }_{j} F_{j i}(\infty)-{ }_{j} F_{j i}(t)\right] d t / \int_{0}^{T}\left[{ }_{i} F_{i j}(\infty)-{ }_{i} F_{i j}(t)\right] d t
$$

approach a limit, finite or $+\infty$, as $T \rightarrow+\infty$.

In particular, if the distributions are in the domain of attraction of a positive stable distribution it is necessary and sufficient that

$$
\left[{ }_{j} F_{j i}(\infty)-{ }_{j} F_{j i}(t)\right] \div\left[{ }_{i} F_{i j}(\infty)-{ }_{i} F_{i j}(t)\right]
$$

approach a limit, finite or $+\infty$, as $t \rightarrow+\infty$ in order that the semiMarkov and dual semi-Markov chains both be ergodic.

5. A relation between the forward and reverse chains. From (8) of $\S 3$, if the chain is normal then

$$
\lim _{n \rightarrow \infty}\left[N_{j j}(n)-N_{i j}(n)\right]={ }_{j} N_{i i i} N_{i j} \lim _{n \rightarrow \infty} P\left[Z_{n}=i \mid Z_{0}=j\right] .
$$

By observing that

$$
{ }_{j} N_{i i} N_{i j}=\left(\alpha_{j} / \alpha_{i}\right){ }_{j} N_{i i}={ }_{i} N_{j j}
$$

cf. [3, p. 247], and comparing with Theorem 9-26 of [3] we identify

$$
\lim _{n \rightarrow \infty} P\left[Z_{n}=i \mid Z_{0}=j\right]=\lim _{n \rightarrow \infty} \sum_{k} \hat{p}_{m k}(n)_{i} \hat{F}_{k j}(\infty) .
$$

From this we are able to conclude the intuitively appealing result that the probability in the long run that the last state of the pair $\{i, j\}$ to be visited by the forward chain was $i$ is equal to the probability that in the long run the next state of the pair $\{i, j\}$ to be visited by the reverse chain will be $i$.

The author wishes to thank the referee for pointing out this relationship which was shown here.

\section{REFERENCES}

1. K. L. Chung, Markov chains with stationary transition probabilities, SpringerVerlag, Berlin, 1960.

2. J. G. Kemeny and J. L. Snell, Potentials for denumerable markov chains, Bull. Amer. Math Soc. 67 (1961), 120-122.

3. J. G. Kemeny, J. L. Snell and A. W. Knapp. Denumerable markov chains, Van Nostrand, Princeton, N. J., 1966.

4. Ronald Pyke, Markov renewal processes: definitions and preliminary properties, Ann. Math. Statist. 32 (1961), 1231-1242.

5. James Yackel, Limit theorems for semi-Markov processes, Trans. Amer. Math. Soc. 123 (1966), 402-424.

Purdue University 